\title{
Sistemas de retroalimentación en la construcción de la identidad digital de la Escuela de Sociología. Universidad Nacional José Faustino Sánchez Carrion de Huacho
}

\section{Feedback systems in the construction of the digital identity of the School of Sociology. José Faustino Sánchez Carrion de Huacho National University}

\author{
Arias Martínez Luis Miguel ${ }^{1}$, Brito Vega Wilfredo ${ }^{1}$, Castillo Amado Julio, Zubieta Núñez Filomeno
}

\begin{abstract}
RESUMEN
A partir de marzo de 2016 y como decantación de experiencias previas, se viene desarrollando desde la Escuela de Sociología de la UNJFSC un proceso de apropiación de herramientas digitales que tiene como motivación principal responder a los requerimientos de digitalización de los procesos de licenciamiento y los de la acreditación. Nuestra escuela ha venido construyendo un espacio digital que tiene por nombre "Sociología en la Red". Provee servicios con la finalidad de: fortalecer los procesos académicos que consisten en: provisión de contenidos digitales especializados, facilitar el acceso a información de calidad de última generación, afianzar servicios para licenciamiento-acreditación y crear comunidad académica a partir de la construcción de una "identidad digital" para la Escuela. El proceso de construcción, parte de un proceso de "empoderamiento" que tiene que ver con la "apropiación" de herramientas de los ámbitos digitales como son los "sistemas de gestión de contenidos", estas facilitan la presencia en el mundo digital a personas y grupos que son usuarios comunes de este tipo de recursos. La primera fase del trabajo, está asegurada y ha puesto por el momento a disposición de la audiencia cuatro mil objetos digitales propios de la disciplina, cuenta con presencia sólida en las redes sociales, y explora posibilidades de integrar por un lado sistemas de "referenciación" que permitan el "reúso" de los contenidos y de otro, consolidar sistemas de gestión de los datos para conocer a los usuarios y sus usos preferenciales y necesidades de la comunidad. Esta es una experiencia en curso que articula herramientas de última generación y provee servicios a una comunidad de al menos 1970 suscriptores de la especialidad
\end{abstract}

Palabras Claves: Decantación de experiencias, identidad digital, sistemas de gestión de contenidos.

\section{ABSTRACT}

As of March 2016 and as a decantation of previous experiences, a process of appropriation of digital tools has been developed from the School of Sociology of the UNJFSC whose main motivation is to respond to the requirements of digitalization of the processes of licensing and accreditation. Our school has been building a digital space called "Sociology on the Web". It provides services with the purpose of: strengthening academic processes that consist of: provision of specialized digital content, facilitating access to state-of-the-art quality information, consolidating services for licensing-accreditation and creating an academic community based on the construction of a "digital identity "for the School. The construction process, part of a process of "empowerment" that has to do with the "appropriation" of tools in the digital fields such as "content management systems", these facilitate the presence in the digital world to people and groups that are common users of this type of resources. The first phase of the work, is assured and has for the moment made available to the audience four thousand digital objects of the discipline, has a solid presence in social networks, and explores possibilities of integrating "referencing" systems that allow the "reuse" of content and other, consolidate data management systems to meet users and their preferential uses and community needs. This is an ongoing experience that articulates state-of-theart tools and provides services to a community of at least 1970 specialty subscribers.

Keywords: Decantation of experiences, digital identity, content management systems..

\section{INTRODUCCION}

La identidad digital, es el resultado de actividades que se desarrollan prácticamente a diario en un proceso que tiene entre sus finalidades en primer lugar, el empoderamiento en referencia a los recursos que pueden configurarse a partir de las TIC; en segundo real una voluntad de difusión de los mismos para su apropiación por los miembros de la comunidad, en tercer lugar el de proveer de recursos suficientes a través de los procesos de generación de información para obtención en este caso de la acreditación institucional, y en cuarto lugar, la visualización de herramientas de retroalimentación para el conocimiento de los usuarios, sus actividades, preferencias y necesidades; pretendemos también, generar ontologías de dominio que permitan la inserción de recursos a partir de la referenciación semántica para la clasificación y almacenamiento los mismos, su uso y recuperación, factores estos dos últimos que están en proceso.

Las herramientas que usamos, cuentan con recursos para la retroalimentación- y es posible disponer de estadísticas sobre las visitas, conocer los intereses dominantes de los usuarios y en general disponer de información sobre la marcha de la experiencia, el acceso a contenidos definidos y actividades de los usuarios a partir de los datos estadísticos que las propias herramientas generan de manera automática. .

Un hecho innegable en este momento es la influencia de las -redes sociales- en la evolución de este tipo de experiencias.
La integración de servicios como Facebook, Twitter o Linkedin; si bien aportan mucha visibilidad, constituyen en sí una forma de competencia con la propuesta central de la iniciativa que es "Sociología en la Red". Portal digital de Sociología en la UNJFSC", Los usuarios, se mueven muy cómodos en servicios como Facebook, menos en Twitter y se conforman con pulsar la tecla del "me gusta" con una mayor o menor profundización en los contenidos, actividad esta que constituye no obstante un insumo fundamental para el conocimiento de sus preferencias y necesidades.

Esta que podemos definir como una plataforma digital, sirve igualmente para la vinculación que se establece con nuestros egresados, se les ha propuesto un nuevo espacio de interacción que se soporta en Facebook y que funciona con la intención de mantener con ellos el vínculo institucional al proveerles entre otros servicios: ofertas de empleo, oportunidades de capacitación complementaria, becas y convocatorias para mejorar sus posibilidades académicas y profesionales.

Además estamos en vías de consolidar un equipo suficiente que viene colaborando de diferentes maneras con el desarrollo de la iniciativa, abierta a estudiantes, profesores y administrativos. Proponemos el desarrollo de alternativas de bajo costo, escalables que contribuyan a la alfabetización tecnológica de los concurrentes, a la apropiación de TIC, a la construcción de comunidad, a la consolidación de repositorios institucionales y a la disponibilidad de recursos digitales para la 
profesionalización de los aspirantes en este caso a sociólogos. La idea básica es la del empoderamiento, que posibilite que los concurrentes, mejoren sus capacidades digitales (Aguaded, Martín-Gutierrez, Caldeiro-Pedreira, 2018, pág. 159). Consideramos que experiencias de esta naturaleza, deben ser impulsadas en las unidades académicas. Proponemos una iniciativa de abajo a arriba, que implica la apropiación de tecnologías de la información las que ponen en la mano de usuarios sin capacidades especiales, instrumentos que permiten un desempeño más que aceptable al proveerles de contenidos relevantes para fortalecer sus capacidades académicas y oportunidades de estudio cumpliendo con los requisitos de acreditación.

\section{Antecedentes teóricos y métodos para el abordaje de la experiencia}

La integración de las llamadas TIC en los ámbitos universitarios supone un desarrollo multidimensional y complejo. Se aprecian a nivel institucional al menos dos líneas de intervención, que tienen que ver por un lado con la implantación de sistemas de soporte al trabajo académico y de otro, la provisión de herramientas para la gestión y administración propios de cualquier organización.

Hay que decir que una propuesta como la que venimos desarrollando a nivel interno y con capacidades propias soportada en herramientas de acceso abierto, puede paliar las carencias, evidentes en las instituciones públicas que no dedican partidas ni presupuesto para el desarrollo de servicios digitalizados.

En el ámbito de la administración de lo académico, la situación es distinta. Las diferentes instituciones desarrollan sus propuestas, en ocasiones con recursos muy limitados y gracias al trabajo de servidores públicos que han tenido a bien asumir la tarea del desarrollo de sistemas informáticos de gestión de información administrativa y académica. No hay desarrollos uniformes y cada institución implementa sistemas en la medida de sus posibilidades, que no son muchas. Este es el caso para las herramientas de registro de asistencia, notas y en general lo académico en nuestra casa de estudio.

La situación es paradójica. Incluso en aquellas universidades donde la apuesta por las TIC es consistente, no hay un norte definido. Es probable que los lineamientos emanados de los reglamentos propios del proceso de "licenciamiento" y los de la "acreditación", puedan permitir impulsar en forma sostenida estas labores.

Consideramos que el verticalismo implícito en los modelos de desarrollo, ha truncado en muchos casos el desarrollo de propuestas interesantes generadas desde abajo, y que han podido en su momento contribuir a reducir estas deficiencias con el desarrollo de herramientas, aplicaciones, utilitarios, propuestas que han facilitado procesos de apropiación con la provisión de servicios para la comunidad universitaria.

\section{TIC y universidades}

En una tesis de maestría, Luza (2013), plantea que para sortear el peligro de que la universidad pública pueda aparecer como desfasada, se requiere una decisión estratégica explorando y explotando las posibilidades asociadas a las TIC, tanto en la docencia como en la investigación y en la instrumentación tecnológica para desarrollos futuros. Propone la necesidad de incentivar el uso de las TIC entre los alumnos y la necesidad de abordar el tema de la alfabetización tecnológica tanto de los docentes como de los alumnos en un planteamiento coincidente con el de Bueno de la Fuente y Hernández (2011), como elemento potenciador de las nuevas formas de trabajo docente y de investigación.

Es necesario tomar conciencia de que este campo de actividades cambiante y dinámico y en periodos de tiempo muy cortos y con cambios cada vez más intensos y significativos. A través del tiempo hemos podido constatar actitudes que van desde la indefensión de los alumnos cuando les pedíamos su dirección de correo electrónico hasta ahora, cuando dan lecciones de manejo de redes sociales. Sin embargo usan el correo electrónico como una llave para acceder a éstas. Esto ocurre en una fase en la que el uso del correo ha devenido en muchos casos marginal frente a las redes como sistema de interconexión, desatendiendo otras propuestas de corte más institucional como las plataformas académicas (Aguaded, Martín-Gutierrez, Caldeiro-Pedreira, 2018). Es un momento en el que los estudiantes, se mueven preferente y masivamente en las redes sociales y se requiere ver como adecuar la oferta de servicios a la nueva situación.

Por ello es necesaria articular estrategias que permitan una alfabetización tecnológica uniforme de la comunidad universitaria (Bueno de la Fuente y Hernández, 2011), de manera que los conocimientos tácitos vinculados en muchos casos al uso recurrente de las redes sociales, puedan convertirse en habilidades explícitas, para que profesores y alumnos puedan sentirse bien e ir ganando en su desempeño en el manejo estas herramientas. Pensamos que esto se da por la desigual difusión de las TIC en el campo de la universidad pública peruana, considerando además que muy probablemente la llamada brecha digital existe e implica componentes que sería necesario resolver o atenuar.

Evidentemente y en el momento actual, las cosas cambian mucho por la integración de nuevos instrumentos, dispositivos interactivos, teléfonos inteligentes que ponen en la mano de los usuarios posibilidades inimaginables y además "amistosas". En este sentido, la innovación permanente de la oferta de dispositivos, puede estar contribuyendo a reducir al menos parcialmente el problema de la brecha en sus diferentes expresiones.

Por otra parte también, en la revisión de bibliografía, hemos encontrado trabajos que tienen que ver con los usuarios finales de las TIC en ámbitos institucionales y académicos, los estilos de participación, las actitudes y las respuestas posibles que podemos encontrar en estas experiencias. (Bettoni et al, 2007 y Carnoy et al, 2005).

Nakano (2014), describe el proceso de implantación de propuestas soportadas en TIC, en este caso en la PUCP. Da cuenta de la multiplicidad de instancias que tienen que ver con el desarrollo de este tipo de sistema y propone la integración de los mismos en una especie de "mando único" que estaría a cargo de una implantación, en su opinión, bien orientada.

Presenta un problema similar al nuestro, pero en sentido contrario. Para nosotros el hecho de la descentralización, las oportunidades que brindan los desarrollos horizontales, pueden resultar apropiados, considerando que el desarrollo de áreas temáticas específicas habrá de corresponder a personas de la especialidad correspondiente.

En nuestra modesta opinión y con recursos inexistentes, pensar en una alternativa diferente resulta en este momento de urgencias marcadas por el licenciamiento, inviable; considerando que los emprendimientos en implantación

\footnotetext{
${ }_{1}$ Escuela de Sociología, Facultad de Ciencias Sociales.UNJFSC.

Recibido: 24/01/2019 Aprobado: 11/03/2019
} 
de TIC requerirían la asignación de recursos en forma permanente para garantizar su sostenibilidad. En los ámbitos de la universidad pública con sistemas burocráticos a veces incluso ineficientes, la emergencia de propuestas desde las diferentes unidades académicas puede constituir una alternativa de desarrollo viable, sugerente y oportuno.

Las principales dificultades para la introducción de las TIC en las universidades son: falta de estrategias institucionales, resistencia a usarlas en docencia y costos elevados. Las universidades, deben clarificar su decisión estratégica sobre el uso de las TIC en la docencia y los demás servicios. En particular, cómo usar las TIC para: propuestas de docencia virtual, nuevos colectivos de estudiantes, formación continuada, difusión y gestión de información (Salinas, 2004), distribución de la misma, generación de fuentes documentales y repositorios, acceso a bases de datos, generación de grupos y equipos de interés.

Todos estos elementos se actualizan de alguna manera según los lineamientos establecidos en este momento por la Superintendencia Nacional de Educación Universitaria (SUNEDU) al proponer una batería de servicios que obligatoriamente las instituciones de educación superior deben brindar a sus concurrentes si desean el "licenciamiento", proceso este que no anticipa por otra parte la provisión de los recursos necesarios para la puesta en marcha de los mismos.

Morla (2014) afirma que: “...vemos como el aumento del número de usuarios en línea, define una clara tendencia hacia el incremento de las inversiones en publicidad en línea y el decrecimento de las inversiones en medios masivos (tv, radio). Factores como la edad del público objetivo y la posibilidad de llegar a donde éste pasa su tiempo, la cantidad de plataformas y servicios gratuitos disponibles, y la posibilidad de aprovechar la data sobre la actividad en las redes sociales, sustentan la elección de los medios sociales como campo para la aplicación del marketing digital. Al mix de las "4 P" en marketing tradicional (Product, Price, Position, Promotion)". (p. 95)

Si bien el estudio se refiere muy precisamente al asunto de las estrategias de marketing, se vincula a nuestra propia experiencia, habida cuenta del hecho de que en nuestro caso, también buscamos visibilidad para la Escuela de Sociología de la UNJFSC y que las estrategias que define Morla (2014), la revisión de las redes sociales y la demostración y conocimiento fino del que dispone sobre su funcionamiento son para nuestro caso absolutamente relevantes. Plantea además que las nuevas herramientas soportadas en las TIC han transformado los procesos de acceso al público objetivo. Por ello resalta la necesidad de integrar consistentemente las TIC en el planeamiento estratégico de la institución, proponiendo lo siguiente:

“...se plantea un plan de marketing digital para medios sociales considerando los factores tecnológicos, se desarrollan las estrategias de marketing social para la Universidad y se dan pautas para la aplicación de analíticas, métricas y herramientas de análisis, según los objetivos elegidos. Se sugiere cómo alinear las acciones que ya está desplegando la PUCP en medios sociales". (p. 112).

Hace énfasis que no existen en este momento herramientas que permitan integrar las diferentes fuentes de datos estadísticos y brindar información consistente sobre la evolución de estas propuestas y la receptividad del público. Un problema similar al de la UNJFSC, a pesar de la distancia, pues en nuestro caso, el objetivo no sería únicamente fortalecer la estrategia institucional sino más bien mejorar la oferta de servicios disponibles para el impulso de las dimensiones "académicas" y "científicas".

Esto último, posibilitaría entre otras cosas fortalecer los procesos de profesionalización- de los concurrentes, al proveerles servicios de última generación, con contenidos seleccionados, ajustados a sus necesidades, al perfil profesional definido por la propia Escuela de Sociología.

No queremos trabajar solo la imagen. Ese, es en nuestro caso, un beneficio derivado. No obstante, el acucioso trabajo de Morla (2014) resulta de gran utilidad por las referencias al funcionamiento de las redes sociales que, efectivamente, aportan beneficios y constituyen probablemente en este momento el mejor medio de interconexión con los usuarios y un recurso para mantener viva la relación con ellos.

En referencia a los procesos de integración interinstitucional en la medida en que suponen una ampliación del campo de referencias y la interconexión con otras instituciones de la especialidad; constituyen para nosotros igualmente un objetivo importante. Guerra, (2014) resalta la importancia de la redes universitarias, y más precisamente, "el caso de la Red Peruana de Universidades, como espacios idóneos para crear capacidades en la generación de conocimiento desde un ethos académico" (p. 27). Sus planteamientos coinciden con nuestras propias intenciones, puesto que partimos de la necesidad de aproximar conocimiento a nuestros usuarios, tanto en propuestas para impulsar las dimensiones académicas como para fortalecer las posibilidades de acceso a documentación de calidad, bases de datos especializadas y ampliación de la oferta formativa; todo lo cual hace crecer significativamente.

Guerra (2014), se plantea las características de la distribución de conocimiento en cada una de las redes e insiste en el peligro que puede implicar, en este nuevo contexto neoliberal que ha brindado las bases para el desarrollo de la era de la información, que la esfera económica se imponga sobre la esfera académica en el espacio de las universidades como viene efectivamente ocurriendo con el desembarco de los grandes capitales en el espacio de la educación en sus diferentes niveles.

La transformación de la educación con soporte en las TIC, decimos nosotros, puede transformar el proceso de profesionalización, ampliar las oportunidades y contribuir a la "cosmopolitización" de los estudiantes de la mano con la adscripción a redes especializadas, los contactos y el intercambio académico posibilitado por el uso de estos dispositivos.

Hay sin embargo que hacer referencia al trabajo de Piscitelli, (2005, p. 40) que afirma en toda la primera parte de su trabajo que la presión es muy fuerte de parte de los poderosos, y da cuenta del proceso de colonización también del ciberespacio con la inundación de contenidos en muchos casos irrelevantes, que contribuyen a la espectacularización de la realidad con intenciones manipulatorias.

\section{La provisión de contenidos en experiencias de digitalización}

En referencia a la temática de la provisión, gestión de contenidos y el tratamiento de la información, es resaltable el trabajo de Bueno de la Fuente y Hernández (2011) que para el caso de lo que define como Repositorios Institucionales de Contenidos Educativos (RICE) realiza una revisión de los aportes al conocimiento de los diferentes problemas y obstáculos en la implantación de recursos para la academia a nivel global.

La descripción precisa de la problemática, presentando 
ilustrativamente la situación en la que se da, de una parte, una presión consistente para implantar sistemas de estas características y en muchos casos, incapacidades institucionales para afrontar eficientemente el desafío de la implementar una oferta de servicios sólida y consistente, con permanencia en el tiempo, capacidades para una actualización permanente y la incorporación de recursos y herramientas actualizadas, escalabilidad y el reconocimiento de los cambios permanentes a los que estos procesos están expuestos.

\section{Procesos de empoderamiento}

A partir de finales de los 90 aparecen con fuerza los sistemas de gestión de contenidos (CMS) que constituyen en la actualidad un recurso inapreciable para el desarrollo de prácticas colaborativas en diferentes ambientes, contextos y espacios comunitarios. La definición básica de un CMS, la aporta Barrio (2006):

"Un CMS, hace referencia a herramientas de autor diseñadas con el objeto de proveer, fundamentalmente a los usuarios, de soluciones sencillas y ágiles para la creación de objetos digitales educativos, especialmente (en el caso de la educación) de objetos de aprendizaje y secuencias didácticas".

De alguna manera, los CMS implican la apertura de posibilidades para la colaboración, al permitir una amplia variedad de operaciones que canalizan la intervención de nuevos actores potenciales, contribuyendo a una transformación que desde nuestro punto de vista es radical en los entornos digitalizados.

En este sentido, es relevante el trabajo de Casero-Ripollés, (2018) quien señala cómo es que el entorno digital introduce notables novedades en la distribución de la información política. La principal tiene que ver con la multiplicación de plataformas a través de las cuales circulan los contenidos.

Con la difusión de estos sistemas asistimos en la actualidad a la proliferación de actores que pueden concurrir de esta forma al desarrollo de nuevas experiencias de acción pública, nuevas formas de articulación de trabajo en grupo y determinadas estrategias de distribución de información, relevante para diferentes intereses y actividades, académicas.

La generación de conocimiento es una tarea que las instituciones de educación superior deben asumir sin titubeos. Las TIC constituyen en nuestro tiempo una herramienta fundamental para ese "desarrollo" esquivo, son un instrumento para la articulación de actores sociales pueden contribuir, a generar un clima de confianza pública imprescindible para la generación de bienestar. Contribuyen también a la generación de procesos identitarios y fortalecen la presencia pública de las instituciones, en este caso, las universidades.

La producción de contenidos en "Sociología en la Red" de la UNJFSC

En realidad y merced a la vigilancia tecnológica, el proceso de provisión de contenidos ajustados a las necesidades de la entidad correspondiente, una escuela profesional, una biblioteca especializada o, un curso específico; no tiene por qué ser complicada. Es suficiente con interconectarse con algunos centros de excelencia para garantizar la provisión de contenidos de calidad.

Lo que si es necesario generar con precisión son los criterios de selección pues la difusión de contenidos, en consonancia a la toma de postura institucional en temas sin duda candentes, controversiales, sobre los que es necesario generar conocimiento, habida cuenta que como institución especializada en la observación del devenir político, económico, social, cultural, podemos y en muchos casos tenemos que generar discurso, producir sentido que permita para la comprensión de los procesos en curso.

Evidentemente se requiere también perseverancia ante circunstancias como la ausencia de reconocimientos o incentivos, elementos imprescindibles para asegurar la sostenibilidad de las experiencias.

Paralelamente, la consolidación de las redes sociales y particularmente la importancia que ha alcanzado la red Facebook en Perú, configuran un campo de servicios en continuo crecimiento. Los CMS integran en este momento la posibilidad de interconexión automática con Facebook, Twitter, Linkedin; que son las redes que hemos integrado en principio a nuestras experiencias. Esto facilita además el conocimiento sobre las inquietudes de los concurrentes interconectados, el rescate de contenidos significativos al interior de la comunidad. Colaborar y compartir, quiere esto decir que además de la labor continuada de seguimiento a la emergencia de contenidos digitales significativos, tenemos que estar también atentos a la emergencia de temáticas propias de los concurrentes y por las que estos muestran preferencias o sensibilidad.

Además este tipo de procesos permite incorporar nuevas áreas temáticas tras una concienzuda evaluación, o bien con la observación de las "buenas prácticas" de instituciones sometidas a consulta, convenientemente enlazadas, de las que a partir de estrategias de benchmarking, se puede aprender en un proceso de detección, captura, apropiación y distribución de informaciones nuevas y significativas para los fines propios de la institución que no son otros que los académicos y profesionalizantes que pasan por la generación de capacidades para el análisis crítico de la realidad.

Está claro que hemos construido una comunidad que resulta beneficiada con la distribución de información de calidad. Esto no existe en ninguna otra dependencia de la institución. Es necesario evidenciar que las redes sociales, no son solución para los requerimientos que se plantean en el proceso de Licenciamiento.

Sin embargo, y pendientes de la evolución y el uso, se ha adoptado el Facebook que está ciertamente vinculado a la línea principal de desarrollo de nuestro portal digital "Sociología en la Red" de la UNJFSC el cual enlaza inmediatamente los contenidos difundidos en la página de facebook de "Sociología en la Red" de la UNJFSC. Nuestro sitio en Twitter (@SociologiaenlaR), resulta visualmente atractivo y está captando lentamente usuarios. Esta red, no es ni mucho menos tan popular como Facebook pero brinda ventajas de acceso a información significativa, facilita una interacción directa con distintos usuarios y resulta consecuentemente inapreciable. Igualmente contamos con el servicio Linkedin desde el inicio de la experiencia y en este momento cuenta con 782 suscriptores. Disponemos de los servicios de Google + que facilitan igualmente el acceso a nuestros contenidos y genera también alertas automáticas sobre contenidos.

\section{Los contenidos}

En referencia al Portal "Sociología en la Red" de la UNJFSC, la información se organiza en función del requerimiento para el licenciamiento y la acreditación de la Escuela de Sociología a partir de un proceso de categorización que integra estos títulos:

\section{Categorías:}

Actualidad (2.292), Becas (132), Biblioteca Digital. Novedades 
(298), Conferencias (74), Convocatorias (531), De recursos didácticos (80), Dirección de la Escuela de Sociología(157), Foro Público (3), Galería de docentes (5), Galería fotográfica (95), Gestión de Investigación (8), Hemeroteca (286), Herramientas interactivas (63), La Comunidad (206), La voz de I@s egresad@s (35), Licenciamiento (42), Novedades (171), Oferta de formación (294), Ofertas de Empleo (91), Páginas Libres (25), Política (961), Políticas públicas (737), RENATI Esc de Sociología (2), Reportes (237), Scoop.it (8), Sin categoría (344), Sociología (75), Tendencias (1.204), Transdisplinar (10), Videoteca (473), Visión crítica (268)

Integra elementos demandados para el proceso de licenciamiento y también pretende responder a algunos de los indicadores del proceso de acreditación. La categorización en un principio ha respondido a estos dos procesos. En el mismo orden de cosas, proveemos acceso a un listado de recursos digitales y bases de datos de la especialidad y de acceso abierto que son los siguientes:

\section{Recursos Digitales}

ALICIA, Biblioteca Central de la UNJFSC, Biblioteca de recursos para formulación de emprendimientos. Biblioteca de recursos para formulación de proyectos, Biblioteca Digital, Biblioteca Digital "Carlos Morales Chirito", Directorio de Revistas Especializadas, Herramientas de campo, La Referencia, Open Access Directory, OpenDOAR, Repositiorio Institucional del IEP, Repositorio Digital de la CEPAL, ScienceDirect are open access.

Se pretende igualmente afianzar las vinculaciones institucionales y se han dispuesto enlaces suficientes a recursos propios y externos que garanticen la provisión de información de calidad para los usuarios de la página.

\section{Sitios de interés}

Asociación Internacional de Sociología, Biblioteca Digital "Carlos Morales Chirito", Faustinianos Emprendedores, Observatorio de Políticas, Oficina de Cooperación Técnica y Convenios - UNJFSC, SOCIOLOGÍA: Ciencia del Milenio, Sociología7. Blog colaborativo para una Sociología posible, Unidad de Transferencia, Innovación y Publicaciones de la UNJFSC

Se puede dar cuenta de la evolución de la experiencia. El crecimiento continuado del número de suscriptores. Contar con 1970 seguidores acumulados en las diferentes modelidades de acceso, constituye para nosotros un aliciente y una alegría. Contamos con datos que pueden constituirse en indicadores claros de desempeño. 17,012 visitas entre marzo y diciembre de 2016; 29,351, correspondientes a 2017; 45,397 correspondientes a 2018 que no concluye. Total al 26 de octubre de 2018: 91,768.

Podemos también contar con las estadísticas que provee nuestro fan page de Facebook que detalla los accesos a los diferentes tópicos, los me gusta, los compartir; las impresiones y los horarios preferentes de los usuarios que constituyen también indicadores precisos de los intereses de los usuarios. Por ejemplo, las 840 visitas que recibió la carta de adcripción de nuestra Escuela a la RIIR (Red de Investigación en Imaginarios y Representaciones) en tan solo tres días. Acumula unos centenares de miles de accesos.

Todo ello constituye un ambiente muy estimulante para darle continuidad a nuestras labores.

\section{CONCLUSIONES}

Desde nuestra propia experiencia, constatamos, que es necesario adoptar una actitud vigilante, habida cuenta de las marchas y contramarchas del proceso de integración de las TIC en ámbitos institucionales; proceso que es, por decir lo menos, evidentemente frágil en el Perú, por la ausencia de políticas públicas consistentes en referencia a la denominada "Sociedad de la Información".

Pero podemos decir también, basados en nuestra experiencia, que el proceso que emprendimos, la integración consistente de las TIC, puede contribuir a la alfabetización tecnológica de alumnos y profesores.

También es importante señalar que el trabajo para generar y proveer acceso a información de calidad digitalizada, supone un trabajo permanente que hay que desarrollar con perseverancia y en muchos casos no reconocidos.

Se requieren procedimientos de reconocimiento de labores que se realizan fuera de la vista del público. Hay quienes incluso piensan que éstas son labores de generación automática pero sí requieren la intervención de personas de carne y hueso que ponen en funcionamiento sus competencias y equipos.

Es necesario también darle continuidad a los trabajos en la línea primero de:

integrar mecanismos de registro que van a permitir un conocimiento más fino de los usos preferenciales de los concurrentes así como de sus necesidades y preferencias.

Finalmente, se requiere igualmente integrar sistemas semánticos de referenciación que nos están permitiendo de momento construir una ontología de dominio para la generación de repositorios especializados. La implantación de sistemas de esta naturaleza, facilitará la búsqueda y recuperación de la información disponible en nuestras colecciones que cuentan en este momento con 4,212 objetos digitales.

\section{REFERENCIAS BIBLIOGRAFICAS}

Aguaded, I; Martín-Gutierrez, I; Caldeiro-Pedreira, MC. (2018). Desarrollo de competencia mediática en el contexto iberoamericano. Letral(20), 156-181. doi:http:// dx.doi.org/10.30827/RL.v1j20.7814

Barrio, M.G.(2006) Covergencia multimedia y educación, aplicaciones y estrategias de colaboración en la red. Revista de comunicación y nuevas tecnologías 14 (7);1-7.

Bettoni, Marco C., et alts. (2007). Knowledge Cooperation in Online Communities: a Duality of Participation and Cultivation. The Electronic Journal of Knowledge Management. Volume 5 Issue 1 200, 1-6. Recuperado el 20 de 11 de 2016, de http://citeseerx.ist.psu.edu/viewdoc/ download?doi=10.1.1.476.2247\&rep=rep1\&type=pdf

Bueno de la Fuente, G., \& Hernández Pérez, T. (junio de 2011). Estrategias para el éxito de los repositorios institucionales de contenido educativo en las bibliotecas digitales. bid. Textos Universitaris de Biblioteconomía y Documentació. doi:ISSN 15755886

Carnoy, M. (2005). Las TIC en la enseñanza: posibilidades y retos. Lección inaugural del año académico 2004-5. Barna: Universitat Oberta de Catalunya. Obtenido de http://www.uoc.edu/inaugural04/dt/esp/carnoy1004. pdf 
Casero-Ripollés, A. (2018). Investigación sobre información política y redes sociales: puntos clave y retos de futuro. El profesional de la información, 27(5), 964974. doi:https://doi.org/10.3145/epi.2018.sep.01

Guerra Caminiti, E. (2014). Las redes que crean capacidades: el caso de la Red Peruana de Universidades. PUCP, Escuela de Posgrado. Política y Gestión Universitaria. Lima: PUCP. Recuperado el 19 de 11 de 2016, de http://tesis.pucp.edu.pe/repositorio/ handle/123456789/5687

Luza Montero, C. (2013). "Un modelo de arquitectura de información basado en el Balanced Scorecard para el control de la Gestión Estratégica Universitaria". Ingeniería de Sistemas y Telecomunicaciones. Lima: Escuela de Posgrado - UIGV.

Morla Chiong Konfook, Cristina Kim. (2014). Marketing en medios sociales para una institución de educación superior. PUCP, Política y Gestión Universitaria.
Lima: Escuela de Posgrado. Recuperado el 18 de 11 de 2016, de http://tesis.pucp.edu.pe/repositorio/ handle/123456789/5690

Nakano Osores, T. (2014). "Integración y gobernanza de las TIC en universidades. Análisis situacional de la PUCP". Escuela de Posgrado - Pucp. Lima: Maestría en Política y Gestión Universitaria. Recuperado el 26 de 11 de 2016, de file:///H:/Maestria\%20 2016/Taller\%20investigación\%20de\%20Maestría/ Tesis\%20externas/NAKANO_OSORES_TERESA INTEGRACION_TIC\%20en\%20universidades.pdf

Piscitelli, A. (2005). Internet la imprenta del Siglo XXI. Barcelona: Gedisa.

Salinas, J. (2004). Innovación docente y uso de las TIC en la enseñanza universitaria. Revista de Universidad y Sociedad del Conocimiento (RUSC), 1(1). Recuperado el 25 de 10 de 2018, de http://www. redalyc.org/pdf/780/78011256006.pdf 